Article

\title{
Changes in Antioxidants and Sensory Properties of Italian Chocolates and Related Ingredients Under Controlled Conditions During an Eighteen-Month Storage Period
}

\author{
Arianna Roda and Milena Lambri *(D) \\ DiSTAS-Department for Sustainable Food Process, Università Cattolica del Sacro Cuore, 29122 Piacenza, Italy; \\ arianna.roda@unicatt.it \\ * Correspondence: milena.lambri@unicatt.it; Tel.: +39-(0)523-599229; Fax: +39-(0)523-599232
}

Received: 24 August 2019; Accepted: 29 October 2019; Published: 9 November 2019

check for updates

\begin{abstract}
Background: While there has been an increasing interest in the health properties of chocolate, limited research has looked into the changes of antioxidants occurring in the time span from production to the best before date, which was a period of 18 months in this study. Methods: Humidity, ash, pH, acidity, fiber, carotenoids, retinols, tocopherols, sugars, proteins, theobromine, caffeine, polyphenols, fats, the peroxide value, organic acids, and volatile compounds, along with the sensory profile, were monitored at 18-week intervals for 18 months under conditions simulating a factory warehouse or a point of sale. Results: At the end of the storage period, more polyphenols were lost $(64 \%$ and $87 \%)$ than vitamin $\mathrm{E}(5 \%$ and $14 \%)$ in cocoa mass and cocoa powder, respectively. Conversely, a greater loss in vitamin E (34\% and $86 \%)$ than in polyphenols (19\% and $47 \%$ ) was shown in the hazelnut paste and gianduja chocolate, respectively. The sensory profiling of cocoa mass, cocoa powder, and hazelnut paste revealed increases in grittiness and astringency, as well as decreases in melting, bitterness, and toasted aroma. Moreover, in the hazelnut paste and gianduja chocolate, oiliness increased with a toasted and caramel aroma. Furthermore, dark chocolate was more gritty, acidic, and bitter. Milk chocolate lost its nutty aroma but maintained its sweetness and creaminess. Conclusions: These results should contribute an important reference for companies and consumers, in order to preserve the antioxidants and understand how antioxidants and sensory properties change from the date of production until the best before date.
\end{abstract}

Keywords: Italian chocolate; quality; cocoa-based ingredients; monitoring; nutrition

\section{Introduction}

Many daily consumed foods, such as fruits, vegetables, wine, coffee, and chocolate, are attracting increasing attention due to their potential health effects thanks to their richness in polyphenols $[1,2]$. Recent studies have focused on cocoa products commonly consumed for pure pleasure, since they also generate tangible benefits for human health [3]. Indeed, cocoa is a complex product with over 300 constituents [4] and one of the richest sources of flavanols [5]. Cocoa belongs to the "nervine food group" because it contains xanthine alkaloids, with the most important being theobromine ( $2 \%)$ and caffeine [6]. When eaten in moderation as part of a balanced diet, it has been suggested that flavanols from cocoa products may exert beneficial effects on the cardiovascular risk via effects of lowering the blood pressure, anti-inflammation, antiplatelet function, higher HDL, and decreased LDL oxidation. This evidence has been supported by a systematic review of 136 publications [7] and by the European Food Safety Agency [8], which stated a health claim for dark chocolate with a high flavanol content due to its impact on "maintenance normal endothelium-dependent vasodilation which contributes 
to normal blood flow". In order to obtain the claimed effect, $200 \mathrm{mg}$ of cocoa flavanols should be consumed daily. This amount could be provided by $2.5 \mathrm{~g}$ of high-flavanol cocoa powder or $10 \mathrm{~g}$ of high-flavanol dark chocolate.

On the other hand, the popularity of hedonic foods, like chocolate, mostly depends on its sensory properties, which in turn constitute the key for the acceptance of food products on the market. Consumers measure the quality and take raw information on the composition while evaluating the appearance, aroma, texture, taste, and flavor $[9,10]$. Additionally, experienced consumers have their own capability to search for defects leading to the rejection of chocolate and often caused by factors such as the temperature, relative humidity, and light [11], which in turn affect the stability of cocoa butter $[9,12,13]$. Indeed, the fat components are the main components responsible for oxidative deterioration which leads to the formation of off-flavors in chocolates $[9,14]$.

Although there has been an increasing interest in the health properties of chocolate, mainly due to its polyphenol content, limited research has looked into the chocolate matrix to investigate the changes in polyphenols, vitamins, fats, and volatile compounds which also determine the quality [15-17]. While consumers are increasingly demanding high-quality food, they expect this quality to be maintained until the consumption time, but little is known about the evolution of chocolates and related raw materials in response to the storage conditions in a factory warehouse, as well as at a point of sale. The present study aims to monitor Italian chocolates and related ingredients for their polyphenol concentration, vitamin E, peroxide, and acidity values during storage for eighteen months under controlled conditions simulating a factory warehouse or a point of sale. In this study, sensory analysis was considered to outline the chemical changes which produce wider variations, so is more interesting from a commercial point of view.

\section{Materials and Methods}

\subsection{Samples}

Three types of chocolate (dark (D), milk (M), and gianduja (G)) and three ingredients (cocoa mass in solid form (CM), cocoa powder 22-24 (C), and hazelnut paste (HP)) were supplied by Venchi S.p.A. (Castelletto Stura, Cuneo, Italy). The recipes of the chocolates were as follows:

- D: cocoa 56\% min., cocoa mass, sugar, cocoa butter, emulsifier: soy lecithin, and natural vanilla flavor;

- M: cocoa $31.8 \%$ min, milk: $23.5 \%$ min., sugar, whole milk powder, cocoa butter, cocoa mass, anhydrous milk fat, emulsifier: soy lecithin, and natural vanilla flavor;

- G: cocoa $21.2 \%$ min., Piedmont hazelnut pasta I.G.P. (33\%), sugar, whole milk powder, cocoa butter, cocoa mass, anhydrous milk fat, emulsifier: soy lecithin, and natural vanilla flavor.

All the samples were collected immediately after production $(\mathrm{t} 0)$ and stored in their own packages in an air-conditioned room at $21 \pm 2{ }^{\circ} \mathrm{C}$ and $65 \%$ Relative Humidity (RH) for eighteen months, i.e., the best before date established by the company within the EU Regulation 2073:2005 [18]. An eighteen-week interval was decided for sampling considering both the number of samples made available by Venchi S.p.A and the storage time ( $\mathrm{t} 1, \mathrm{t} 2, \mathrm{t} 3$, and $\mathrm{t} 4)$.

\subsection{Microbiological Analysis}

The total microbial count (bacteria, fungi/molds, and yeasts) according to International Standard Organization (ISO) 4833-1:2013 [19], along with Enterobacteriaceae and coliforms as respectively described in ISO 21528-1:2017 [20] and ISO 4832:2006 [21], were detected at the production time (t0) and at the end of storage ( $t 4)$. The number of colony-forming units (CFU) per gram of dry mass of the sample was provided. 


\subsection{Chemical Analysis}

\subsubsection{Humidity, Ash, pH, Acidity, and Fiber}

Humidity and ash were determined according to Association of Official Analytical Chemists (AOAC) 931.04 [22], while food and raw fiber were analyzed using the AOAC 985.29 [23] methodology. Total acidity and $\mathrm{pH}$ were analyzed as reported in the official methods of the Office International du Cacao et du Chocolat et de la Confiserie [24]. For both analyses, a pH meter (CRISON MICRO TT 2050, Carpi, Modena, Italy) was used. The $\mathrm{pH}$ of a $10 \mathrm{~g}$ sample dissolved in $90 \mathrm{~mL}$ of boiling distilled water was measured. The acidity was dosed in the same solution with $20 \mathrm{~mL}$ of sodium hydroxide $0.1 \mathrm{~N}$ and then potentiometrically titrated with hydrochloric acid $0.1 \mathrm{~N}$ until pH 7.0. The result was finally expressed in mg equivalents of stearic acid in a $100 \mathrm{~g}$ sample.

\subsubsection{Carotenoids, Retinol, Tocopherols, and Sugars}

Carotenoids and retinol were determined by applying the AOAC 941.15 method [25], while tocopherols were dosed in compliance with Calvo et al. [26] and Belšcak et al. [27]. The chromatographic determination of tocopherols was performed on an HPLC system, including a Perkin Elmer (Norwalk, CT, USA) 200 Series pump equipped with a Perkin-Elmer 650-10S fluorescence detector, Jasco LC-Net II/ADC (Oklahoma City, OK, USA) communication module, and ChromNAV Control Center software. A LiChrosorb Si60-5 C18 column $250 \mathrm{~mm} \times 4.6 \mathrm{~mm}, 5 \mu \mathrm{m}$ (Supelco, Bellefonte, PA, USA) was used, the mobile phase was hexane:isopropanol:ethanol (98.5:1:0.5) at a flow rate of $1.0 \mathrm{~mL} / \mathrm{min}$ and the injection volume was $20 \mu \mathrm{L}$. The fluorescence detector was set at $290 \mathrm{~nm}$ excitation and $330 \mathrm{~nm}$ emission wavelengths. $\alpha_{-}, \gamma_{-}$, and $\delta$ - tocopherols were identified by comparing the retention times with those of commercial standards. The results were expressed as mg of Vitamin E including $\alpha_{-}^{-}, \gamma_{-}^{-}$, and $\delta_{-}$ tocopherols contained in $100 \mathrm{~g}$ of dry sample. To analyze reducing and non-reducing sugars in cocoa liquor and hazelnut paste, the Luff-Schoorl volumetric analysis suggested by Balestrieri and Marini [6] was adopted.

\subsubsection{Proteins}

Protein determination was carried out following AOAC 939.02-1939 [28], as follows. Two grams of sample were dried in a stove for $24 \mathrm{~h}$. Thirty milliliters of concentrated sulfuric acid (96\%) were added to the sample and homogeneously dispersed, and the mixture was subsequently put into the digester (K-424 BUCHI) until complete digestion. Then, distillation was performed using a semi-automatic system (VELP SCIENTIFICA UDK R7), which adds $90 \mathrm{~mL}$ of sodium hydroxide (32\%) and collects the distilled ammonia in $50 \mathrm{~mL}$ of boric acid $(40 \mathrm{~g} / \mathrm{L})$, to which $0.5 \mathrm{~mL}$ of mixed indicator methyl red/bromocresol green was added. Finally, titration with $0.1 \mathrm{~N}$ sulfuric acid was carried out.

\subsubsection{Theobromine, Caffeine, and Polyphenols}

For determining theobromine, caffeine, and polyphenols, samples were defatted by extracting $50 \mathrm{~g}$ of each sample three times with $250 \mathrm{~mL}$ of $n$-hexane and drying the resulting powder under a nitrogen stream to remove the residual organic solvent [29]. For determinations of caffeine and theobromine, the powder was dispersed in methanol before spectrophotometric analysis, which was carried out by means of a Lambda Bio 40 UV-VIS spectrometer (Perkin Helmer).

The theobromine was determined following López-Martinez et al.'s method [30]. Since the maximum theobromine absorption in our samples was observed at $240 \mathrm{~nm}$, a calibration curve with methanolic solutions containing 5, 10, 15, 20, 25, 30, and $35 \mathrm{mg} / \mathrm{L}$ of theobromine was produced at $240 \mathrm{~nm}$.

Caffeine was determined according to the procedure described by Hečimović et al. [31]. The absorbance was read at $274 \mathrm{~nm}$ and a calibration curve was built using solutions at 20, 40, 60, 80, and $100 \mathrm{mg} / \mathrm{L}$ of caffeine dissolved in methanol. 
The total polyphenol content was determined for the defatted samples dispersed in distilled water according to Belšcak et al. [27], and the absorbance was recorded at $765 \mathrm{~nm}$ using a Shimadzu UV-1601 spectrophotometer (Shimadzu Europe, Duisburg, Germany). Gallic acid was used as a standard in a calibration curve with solutions of $20,40,80,100$, and $120 \mathrm{mg} / \mathrm{L}$ of gallic acid. The results were expressed as mg gallic acid equivalents (GAE) per $100 \mathrm{~g}$ of defatted sample.

\subsubsection{Fat Content}

To determine the fat content, the AOAC Official Method [25], modified as follows, was used. The samples initially underwent acid hydrolysis and extraction by means of a Soxhlet device. Different quantities of sample were weighed, including 3-4 g of cocoa liquor and hazelnut paste, and $4-5 \mathrm{~g}$ of cocoa powder. These quantities were mixed with $45 \mathrm{~mL}$ of distilled water at boiling point, after which $55 \mathrm{~mL}$ of hydrochloric acid $(25 \% w / v)$ was added. The solutions boiled for about $30 \mathrm{~min}$ with a reflux condenser and were then filtered with a Whatman $n^{\circ} 595 \frac{1}{2}$ filter. The filter containing the hydrolyzed sample was thoroughly washed with distilled water until the chloride vanished, and it was then dried at $100{ }^{\circ} \mathrm{C}$ for $6 \mathrm{~h}$. Finally, the fat matter was extracted by means of a Soxhlet device, where the dry filter underwent extraction with $50 \mathrm{~mL}$ of $n$-hexane for $4 \mathrm{~h}$. The fatty acid and sterol profile were determined according to EC Regulation 2568/91 [32].

\subsubsection{Peroxide Value}

To separate the lipid fraction, $50 \mathrm{~g}$ of each sample was extracted three times with $250 \mathrm{~mL}$ of $n$-hexane [29]. The resulting mixture was centrifuged at $3000 \mathrm{rpm}$ for $15 \mathrm{~min}$ (Varifuge 20 RS Hereaus Sepatech, Hanau, Germany), and the hexane was removed using Rotavapor (Büchi Rotavapor R-114, Flawil, Switzerland). The oil recovered was analyzed to determine the peroxide value using the method reported in the EC Regulation 2568/91 [32].

\subsubsection{HPLC Analysis of Organic Acids}

Twenty grams of sample were melted in $200 \mathrm{~mL}$ of hot distilled water, and then decolorized by means of a suitable quantity of carbon powder. The mix was centrifuged at $5000 \mathrm{rpm}$ for $15 \mathrm{~min}$ at $5{ }^{\circ} \mathrm{C}$ (Centrifuge SL 16 R Thermo Scientific), with subsequent filtration by Whatman $n^{\circ} 589 / 3$ filters with a porosity of $150 \mu \mathrm{m}$. A collected volume of $30-35 \mathrm{~mL}$ was acidified with sulfuric acid $(25 \% \mathrm{w} / \mathrm{v})$ until reaching a pH 2.5. The acidified solution was centrifuged at $8000 \mathrm{rpm}$ for $15 \mathrm{~min}$ at $15^{\circ} \mathrm{C}$ (Varifuge $20 \mathrm{RS}$ Hereaus Sepatech), in order to further purify the extract from any sediments, pigments, or turbidity. The supernatant obtained was filtered by means of $45 \mu \mathrm{m}$ syringe filters. HPLC (Spectra system P4000) conditions were as follows: stationary phase Phenomenex Rezex column ROA-ORGANIC ACID H+ at $40^{\circ} \mathrm{C}$; mobile phase $0.005 \mathrm{~N}$ sulfuric acid solution with a flow of $0.5 \mathrm{~mL} / \mathrm{min}$ and a $20 \mu \mathrm{L}$ injection volume; Spectra system UV 1000 detector at $210 \mathrm{~nm}$.

\subsubsection{GC Analysis of the Volatile Compounds}

Chocolates and related ingredients' samples were first dispersed in MilliQ water heated at $80^{\circ} \mathrm{C}$, and then centrifuged at $5000 \mathrm{rpm}$ for $15 \mathrm{~min}$ at $15^{\circ} \mathrm{C}$ (Centrifuge SL $16 \mathrm{R}$ Thermo Scientific) and filtered (Whatman $n^{\circ} 595 \frac{1}{2}$ ). Before extraction, magnesium sulfate was added in order to minimize emulsions. Then, $1 \mathrm{~mL}$ of sample was substituted with the same volume of internal standard (1-heptanol) at a concentration of $50 \mathrm{ppm}$. Afterwards, $500 \mathrm{~mL}$ of sample was extracted for $6 \mathrm{~h}$ with the solvent mixture pentane:dichloromethane (2:1). During extraction, the sample was maintained at $60^{\circ} \mathrm{C}$. At the end it was purified with the addition of anhydrous sodium sulphate and then concentrated by means of Rotavapor (BUCHI Rotavapor R-114) up to a volume of $1 \mathrm{~mL}$. For the determination of volatile compounds, gas chromatography (Autosystem XL Gas Chromatograph Perkin Helmer) with a Flame Ionization Detector (FID) was used, following the chromatographic conditions indicated by Bonvehì [33], by injecting $1 \mu \mathrm{L}$ of extract. Standard solutions were prepared with pentane:dichloromethane (2:1) as the solvent. 


\subsection{Sensory Analysis}

In order to allow a complete sensory description of products and to identify key sensory attributes of chocolates and related ingredients [11] the Quantitative Descriptive Analysis (QDA) [34,35] was applied as a suitable procedure for an assessment of the sensory quality of chocolates and related ingredients during the whole period of storage. No approval from the Human Ethics Committee was required by our institution to perform the sensory analysis in this research.

\subsubsection{Determination of the Sensory Profile of Chocolates and Related Ingredients}

Sensory properties of the chocolates and related ingredients were monitored at each control time ( $\mathrm{t} 0, \mathrm{t} 1, \mathrm{t} 2, \mathrm{t} 3$, and $\mathrm{t} 4)$ under conditions and procedures compliant to ISO standards [36-38]. The QDA was carried out using eight assessors with a broad experience in sensory evaluation, as well as interest and availability. The training was performed as reported by Donadini, Fumi, and Lambri [39]. The QDA was composed of the following stages [34,35]: (1) a lexicon generation process and (2) a set of sensory tests designed to quantify the intensity of the sensory descriptors established in the lexicon generation phase on a rating scale. The chocolate and ingredient samples were prepared and individually served to panelists. Three-digit random numbers were assigned to each sample for tracking purposes prior to service. The order of presentation was balanced and randomized across samples, panelists, and replicates, according to a rotated tasting plan [40].

\subsubsection{Lexicon Generation Process}

In the lexicon generation process, participants were preliminarily asked to name as many sensory characteristics as possible [41-43] which they considered important for the descriptive evaluation of chocolate [44-48]. Redundant terms were discussed openly, with the intervention of the panel leader as a moderator. Descriptors cited by at least $30 \%$ of the panel were retained and intensively discussed among panelists in an open session until agreement was reached on the final verbal definition. Selected sensory attributes as defined for each sample are reported in Table 1.

Table 1. Descriptors used for sensory profiling.

\begin{tabular}{|c|c|c|c|}
\hline Attribute & Description & Range and References & Sample(*) \\
\hline Brightness & $\begin{array}{l}\text { Ability to reflect light; luminescence } \\
\text { of color, with descriptions ranging } \\
\text { from dull to shiny [42] }\end{array}$ & $\begin{array}{c}\text { Low: Dull; dark chocolate } 90 \% \\
\text { cocoa with fat blooming } \\
\text { High: Shiny; dark chocolate } \\
90 \% \text { cocoa }\end{array}$ & $\mathrm{D}-\mathrm{M}-\mathrm{G}$ \\
\hline Snap & $\begin{array}{l}\text { The noise and force with which the } \\
\text { sample breaks or fractures [39] }\end{array}$ & $\begin{array}{l}\text { Low: Gianduja chocolate } \\
\text { High: Dark chocolate } 90 \% \\
\text { cocoa }\end{array}$ & $\mathrm{D}-\mathrm{M}$ \\
\hline Firmness & $\begin{array}{l}\text { Force required for compressing the } \\
\text { sample between molar teeth [45] }\end{array}$ & $\begin{array}{l}\text { Low: Milk chocolate } \\
\text { High: Dark chocolate } 90 \% \\
\text { cocoa }\end{array}$ & $\mathrm{MC}-\mathrm{D}-\mathrm{M}$ \\
\hline Crunchiness & $\begin{array}{c}\text { Easily broken or ruptured } \\
\text { Degree to which the sample } \\
\text { fractures into pieces on the first bite } \\
\text { with the molars [42] }\end{array}$ & $\begin{array}{l}\text { Low: Dark chocolate } 90 \% \\
\text { cocoa } \\
\text { High: Milk chocolate }\end{array}$ & MC \\
\hline Melting & $\begin{array}{c}\text { Chocolate property of melting in } \\
\text { mouth while chewing [45] till } \\
\text { liquefaction [46] }\end{array}$ & $\begin{array}{c}\text { Low: Dark chocolate } 70 \% \\
\text { cocoa warmed in a microwave } \\
\text { oven during } 20 \mathrm{~s} . \\
\text { High: Dark chocolate } 70 \% \\
\text { cocoa warmed in a microwave } \\
\text { oven during } 40 \mathrm{~s} .\end{array}$ & $\mathrm{MC}-\mathrm{C}-\mathrm{HP}-\mathrm{D}-\mathrm{M}-\mathrm{G}$ \\
\hline Stickiness & $\begin{array}{l}\text { The degree a sample sticks to the } \\
\text { palate }[42,47]\end{array}$ & & $\mathrm{MC}-\mathrm{HP}-\mathrm{D}-\mathrm{M}-\mathrm{G}$ \\
\hline
\end{tabular}


Table 1. Cont.

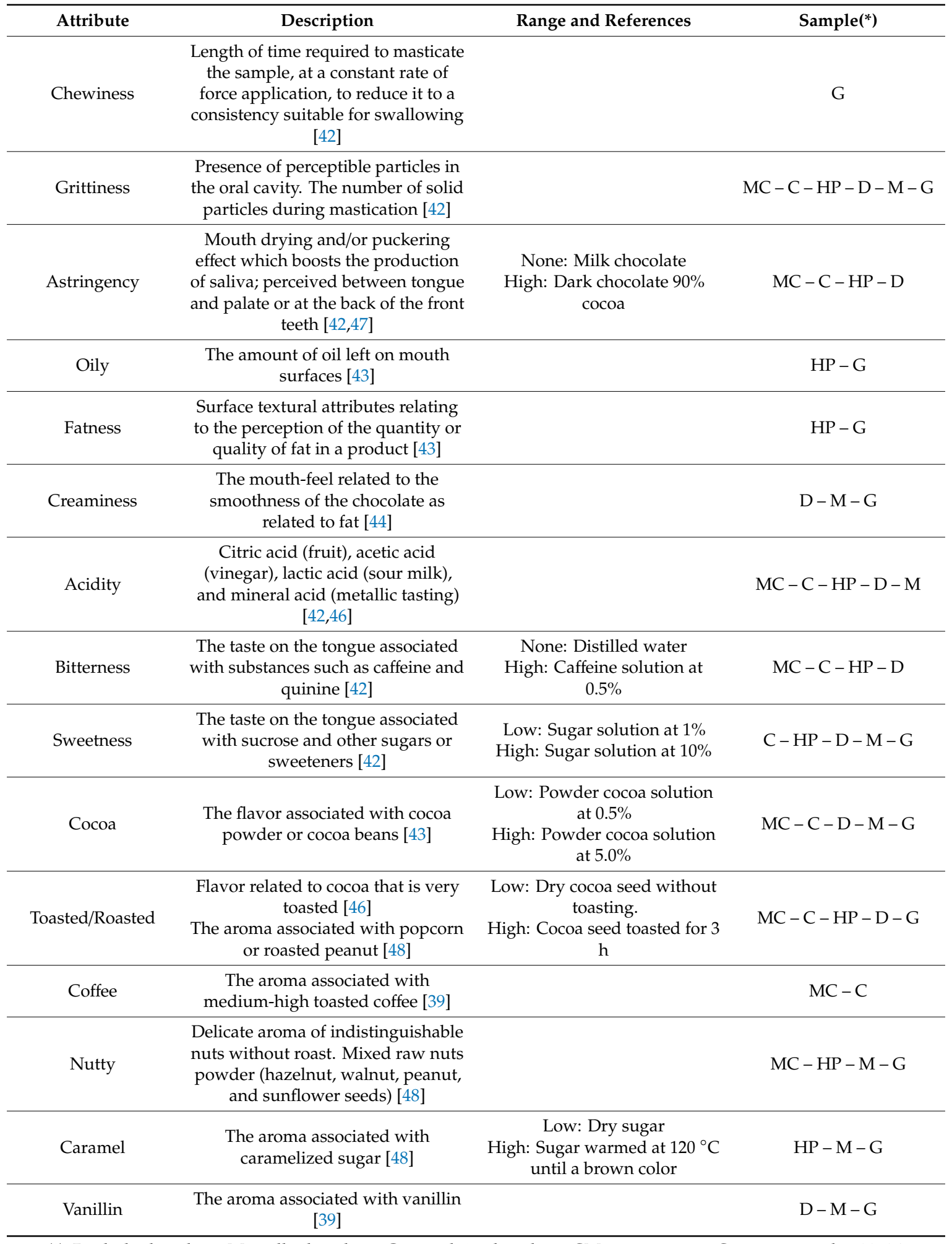

$\left({ }^{*}\right) \mathrm{D}=$ dark chocolate; $\mathrm{M}=$ milk chocolate; $\mathrm{G}=$ gianduja chocolate; $\mathrm{CM}=$ cocoa mass; $\mathrm{C}=$ cocoa powder $22-24$; $\mathrm{HP}=$ hazelnut paste.

\subsubsection{Sensory Tests of Chocolates and Ingredients to Rate Sensory Attributes}

A set of sensory tests was designed to quantify the intensity of the sensory descriptors that were inserted into the score card on a 9-point scale (anchored at both extremes as "not perceived at all" and 
"extremely intense"). Panelists were provided with mineral water and unsalted breadsticks to cleanse their palates between samples.

\subsection{Statistical Analysis}

At each storage time starting from production, the sampling proceeded with two independent replications and each analysis was performed in triplicate. Data were subjected to Microsoft Excel 2017 and to Levene's test to point out the homogeneity of variance among sample subsets. Furthermore, data were analyzed by one-way analysis of variance (ANOVA) with Tukey's $t$-test at $p \leq 0.05$ to highlight the significance of the differences among the different storage times $(t 0, t 1, t 2, t 3$, and $t 4)$ for equal sample types (CM, C, HP, D, M, and G). The statistics were prepared using IBM SPSS Statistics 20 (IBM Corporation, New York, NY, USA).

\section{Results and Discussion}

\subsection{Microbiological Analysis}

The results of the microbiological analysis were compliant with the limits stated by EC Regulation 2073/2005 [18], as reported in Table 2. Different to other studies focused on microbial evolution in chocolate-based and confectionery products [49], in this study, no meaningful data were obtained. This outlined the microbiological quality of the samples under study, along with the strong hygienic conditions applied in production and storage.

Table 2. Results from microbiological analysis at the end of storage.

\begin{tabular}{ccccccc}
\hline Parameter & CM & C & HP & D & M & G \\
\hline Total microbial count & $<5000$ & $<5000$ & $<5000$ & $<5000$ & $<5000$ & $<5000$ \\
Enterobacteriaceae & n.d. & n.d. & n.d. & n.d. & n.d. & n.d. \\
Coliforms & n.d. & n.d. & n.d. & n.d. & n.d. & n.d. \\
\hline
\end{tabular}

n.d. = not detectable

\subsection{Nutritional Composition and Related Characterization}

The sample characterization at $\mathrm{t} 0$ along with phenolic compounds, alkaloids, and vitamins is shown in Table 3, while determination of the fatty acid profile, fatty acid composition, and sterol composition is detailed in Table 4. Finally, the organic acids and the volatile compounds are reported in Tables 5 and 6, respectively. 
Table 3. Characterization at $\mathrm{t}(0)$ along with phenolic compounds, alkaloids, and vitamins analyzed in cocoa mass (CM), cocoa 22-24 (C), hazelnut paste (HP), dark chocolate (D), milk chocolate $(\mathrm{M})$, and gianduja chocolate $(\mathrm{G})$. Data represent the mean $\pm \mathrm{SD}(n=3)$. Within each row, different superscript letters indicate statistically different values among samples according to a post-hoc comparison (Tukey's test) at $p \leq 0.05$.

\begin{tabular}{|c|c|c|c|c|c|c|}
\hline Parameter & $\mathbf{C M}$ & $\mathrm{C}$ & HP & $\mathbf{D}$ & $\mathbf{M}$ & G \\
\hline Humidity (\%) & $1.06 \pm 0.03^{\mathrm{d}}$ & $2.11 \pm 0.01^{\mathrm{a}}$ & $0.75 \pm 0.01^{\mathrm{e}}$ & $0.70 \pm 0.03^{f}$ & $1.17 \pm 0.09^{c}$ & $1.37 \pm 0.00^{b}$ \\
\hline $\mathrm{pH}$ & $5.51 \pm 0.02 e^{e}$ & $8.31 \pm 0.10^{\mathrm{a}}$ & $5.71 \pm 0.01 \mathrm{~d}$ & $5.70 \pm 0.05^{d}$ & $6.29 \pm 0.04^{b}$ & $6.17 \pm 0.03^{c}$ \\
\hline Acidity (mg eq stearic acid/100 g) & $361.00 \pm 0.01^{\mathrm{a}}$ & n.d. & $159.00 \pm 0.01^{c}$ & $184.91 \pm 2.84^{b}$ & $110.95 \pm 5.69^{\mathrm{e}}$ & $128.02 \pm 2.84^{\mathrm{d}}$ \\
\hline Ash $(\%)$ & $4.37 \pm 0.97^{b}$ & $11.70 \pm 0.04^{\mathrm{a}}$ & $2.05 \pm 0.01^{c}$ & $1.67 \pm 0.03^{\mathrm{e}}$ & $1.71 \pm 0.01^{\mathrm{d}}$ & $1.65 \pm 0.01^{\mathrm{f}}$ \\
\hline Protein $(\%)$ & $14.11 \pm 0.63^{b}$ & $23.33 \pm 0.08^{a}$ & $3.90 \pm 0.18^{f}$ & $7.20 \pm 0.01^{d}$ & $6.40 \pm 0.08^{e}$ & $10.10 \pm 0.41^{\mathrm{c}}$ \\
\hline Fat matter $(\%)$ & $35.70 \pm 0.04^{\mathrm{c}}$ & $17.90 \pm 0.12^{\mathrm{f}}$ & $66.80 \pm 1.75^{\mathrm{a}}$ & $23.20 \pm 0.30 \mathrm{e}$ & $34.20 \pm 1.61^{\mathrm{d}}$ & $37.50 \pm 2.30^{b}$ \\
\hline Total sugar $(\%)$ & $0.80 \pm 0.00^{\mathrm{e}}$ & $1.20 \pm 0.03^{\mathrm{d}}$ & $1.60 \pm 0.57^{\mathrm{d}}$ & $45.60 \pm 0.31^{b}$ & $54.70 \pm 0.25^{\mathrm{a}}$ & $40.80 \pm 1.39^{c}$ \\
\hline Fiber $(\%) *$ & $12.50 \pm 0.06^{b}$ & $28.03 \pm 0.14^{a}$ & $8.83 \pm 0.04^{c}$ & $7.56 \pm 0.04 d$ & $1.60 \pm 0.01^{\mathrm{f}}$ & $5.45 \pm 0.03^{\mathrm{e}}$ \\
\hline * raw fiber $(\%)$ & 11.10 & 13.63 & n.d. & n.d. & n.d. & n.d. \\
\hline Phenols (mg Gallic Acid Equivalents /g) & $2.06 \pm 0.25^{b}$ & $7.29 \pm 0.98^{a}$ & $0.31 \pm 0.07^{\mathrm{e}}$ & $2.12 \pm 0.18^{b}$ & $0.64 \pm 0.17^{\mathrm{d}}$ & $0.99 \pm 0.09^{c}$ \\
\hline Caffeine (mg/100 g) & $65.67 \pm 4.97^{\mathrm{b}}$ & $97.69 \pm 5.46^{\mathrm{a}}$ & $3.40 \pm 0.16^{\mathrm{e}}$ & $21.51 \pm 1.04^{\mathrm{c}}$ & $2.06 \pm 0.93^{f}$ & $7.84 \pm 2.27^{\mathrm{d}}$ \\
\hline Theobromine $(\mathrm{mg} / \mathrm{g})$ & $6.77 \pm 0.68^{b}$ & $10.07 \pm 0.10^{\mathrm{a}}$ & $2.04 \pm 0.37^{\mathrm{d}}$ & $7.28 \pm 0.59^{b}$ & $3.97 \pm 0.12^{c}$ & $3.63 \pm 0.25^{c, d}$ \\
\hline 13-Cis- $\beta$-Carotene (ppm) & $<0.10 \pm 15 \%$ & $<0.10 \pm 15 \%$ & $<0.10 \pm 15 \%$ & $<0.10 \pm 15 \%$ & $<0.10 \pm 15 \%$ & $<0.10 \pm 15 \%$ \\
\hline 9-Cis- $\beta$-Carotene (ppm) & $<0.10 \pm 15 \%$ & $<0.10 \pm 15 \%$ & $<0.10 \pm 15 \%$ & $<0.10 \pm 15 \%$ & $<0.10 \pm 15 \%$ & $<0.10 \pm 15 \%$ \\
\hline All-Trans- $\alpha$-Carotene (ppm) & $<0.10 \pm 15 \%$ & $<0.10 \pm 15 \%$ & $<0.10 \pm 15 \%$ & $<0.10 \pm 15 \%$ & $<0.10 \pm 15 \%$ & $<0.10 \pm 15 \%$ \\
\hline All-Trans- $\beta$-Carotene (ppm) & $0.34 \pm 15 \%$ & $<0.30 \pm 15 \%$ & $<0.10 \pm 15 \%$ & $<0.30 \pm 15 \%$ & $0.47 \pm 15 \%$ & $0.30 \pm 15 \%$ \\
\hline$\beta$-Cryptoxanthin (ppm) & $<0.10 \pm 15 \%$ & $<0.10 \pm 15 \%$ & $<0.10 \pm 15 \%$ & $<0.10 \pm 15 \%$ & $<0.10 \pm 15 \%$ & $<0.10 \pm 15 \%$ \\
\hline Retinol (ppm) & $<0.10 \pm 15 \%$ & $<0.10 \pm 15 \%$ & $<0.10 \pm 15 \%$ & $<5.00 \pm 15 \%$ & $82.00 \pm 15 \%$ & $5.00 \pm 15 \%$ \\
\hline Vitamin E (ppm) & $6.77 \pm 10 \% \mathrm{~d}$ & $9.40 \pm 10 \% c$ & $304.00 \pm 10 \%$ a & $8.20 \pm 10 \% c$ & $6.63 \pm 10 \% d$ & $114.00 \pm 10 \% \mathrm{~b}$ \\
\hline
\end{tabular}


Table 4. Fatty acid profile, fatty acid composition, and sterol composition expressed in $\%$ (as 100 of the total) in cocoa mass (CM), cocoa 22-24 (C), hazelnut paste (HP), dark chocolate (D), milk chocolate (M), and gianduja chocolate $(\mathrm{G})$ at production time $(\mathrm{t} 0)$.

\begin{tabular}{|c|c|c|c|c|c|c|}
\hline Fatty acid / Sterol & $\mathrm{CM}$ & $\mathrm{C}$ & HP & $\mathbf{D}$ & $\mathbf{M}$ & G \\
\hline Saturated fatty acids & 63.90 & 61.29 & 9.49 & 63.87 & 65.23 & 37.67 \\
\hline Monounsaturated fatty acids & 32.95 & 35.34 & 83.17 & 32.69 & 31.60 & 57.19 \\
\hline Polyunsaturated fatty acids & 3.05 & 3.30 & 7.24 & 3.34 & 3.12 & 5.10 \\
\hline Trans-oleic fatty acids & $<0.01$ & $<0.01$ & $<0.01$ & $<0.01$ & 0.64 & 0.21 \\
\hline Trans-linoleic fatty acids & $<0.01$ & $<0.01$ & $<0.01$ & $<0.01$ & $<0.01$ & $<0.01$ \\
\hline Trans-linolenic fatty acids & $<0.01$ & $<0.01$ & $<0.01$ & $<0.01$ & $<0.01$ & $<0.01$ \\
\hline Trans-palmitoleic fatty acids & $<0.01$ & $<0.01$ & $<0.01$ & $<0.01$ & $<0.01$ & $<0.01$ \\
\hline C4:0 Butyric & $<0.01$ & $<0.01$ & $<0.01$ & $<0.01$ & 0.69 & 0.23 \\
\hline C6:0 Capronic & $<0.01$ & $<0.01$ & $<0.01$ & $<0.01$ & 0.43 & 0.13 \\
\hline C7:0 Enantiic & $<0.01$ & $<0.01$ & $<0.01$ & $<0.01$ & $<0.01$ & $<0.01$ \\
\hline C8:0 Caprylic & $<0.01$ & $<0.01$ & $<0.01$ & $<0.01$ & 0.28 & 0.10 \\
\hline C10:0 Capric & $<0.01$ & $<0.01$ & $<0.01$ & $<0.01$ & 0.58 & 0.22 \\
\hline C10:1 Caproleic & $<0.01$ & $<0.01$ & $<0.01$ & $<0.01$ & 0.07 & $<0.05$ \\
\hline C12:0 Lauric & $<0.01$ & $<0.01$ & $<0.01$ & $<0.01$ & 0.71 & 0.32 \\
\hline C12:1 Lauroleic & $<0.01$ & $<0.01$ & $<0.01$ & $<0.01$ & $<0.05$ & $<0.05$ \\
\hline C13:0 Tridecanoic & $<0.01$ & $<0.01$ & $<0.01$ & $<0.01$ & $<0.01$ & $<0.01$ \\
\hline C13:1 Tridecenoic & $<0.01$ & $<0.01$ & $<0.01$ & $<0.01$ & $<0.01$ & $<0.01$ \\
\hline C14:0 Myristic & 0.10 & 0.11 & $<0.05$ & 0.21 & 2.34 & 0.98 \\
\hline C14:1 Miristoleic & 0.01 & $<0.01$ & $<0.01$ & $<0.01$ & 0.20 & 0.08 \\
\hline C15:0 Pentadecanoic & $<0.05$ & $<0.01$ & $<0.01$ & 0.05 & 0.26 & 0.12 \\
\hline C15:1 Pentadecenoic & $<0.01$ & $<0.01$ & $<0.01$ & $<0.01$ & $<0.01$ & $<0.01$ \\
\hline C16:0 Palmitic & 26.66 & 27.83 & 6.61 & 25.99 & 26.96 & 16.57 \\
\hline C16:1 Palmitoleic & 0.27 & 0.28 & 0.27 & 0.24 & 0.51 & 0.35 \\
\hline C17.0 Eptadecanoic & 0.22 & 0.23 & $<0.05$ & 0.23 & 0.38 & 0.16 \\
\hline C17:1 Eptadecenoic & $<0.05$ & $<0.05$ & 0.08 & $<0.05$ & 0.09 & 0.08 \\
\hline C18:0 Stearic & 35.67 & 32.08 & 2.68 & 36.15 & 31.53 & 18.10 \\
\hline C18:1 Oleic & 32.68 & 35.06 & 82.68 & 32.40 & 30.73 & 56.60 \\
\hline C18:2 Linoleic & 2.87 & 3.10 & 7.16 & 3.11 & 2.83 & 4.94 \\
\hline C18:3 Linolenic & 0.18 & 0.20 & 0.08 & 0.23 & 0.29 & 0,16 \\
\hline C20:0 Arachic & 1.01 & 0.90 & 0.15 & 1.03 & 0.90 & 0,57 \\
\hline C:20:1 Eicosenoic & $<0.05$ & $<0.05$ & 0.14 & 0.05 & $<0.05$ & 0.08 \\
\hline C22:0 Behenic & 0.17 & 0.14 & 0.05 & 0.17 & 0.16 & 0.11 \\
\hline C22:1 Erucic & $<0.01$ & $<0.01$ & $<0.01$ & $<0.01$ & $<0.01$ & $<0.01$ \\
\hline C22:0 Lignoceric & 0.07 & $<0.01$ & $<0.05$ & 0.09 & 0.08 & 0.06 \\
\hline Cholesterol & 1.00 & 1.00 & 0.30 & 1.30 & 26.80 & 14.70 \\
\hline Brassicasterol & $<0.10$ & $<0.10$ & $<0.10$ & $<0.10$ & $<0.10$ & $<0.10$ \\
\hline 2,4-methylene cholesterol & 0.30 & 0.50 & 0.10 & 0.20 & 0.20 & 0.20 \\
\hline Campesterol & 9.00 & 9.40 & 4.10 & 9.60 & 7.20 & 6.60 \\
\hline Campestanol & 0.20 & 0.30 & 0.40 & 0.20 & 0.10 & 0.20 \\
\hline Stigmasterol & 25.80 & 26.10 & 1.20 & 24.70 & 17.80 & 13.90 \\
\hline Delta-7-campesterol & $<0.10$ & $<0.10$ & $<0.10$ & $<0.10$ & $<0.10$ & $<0.10$ \\
\hline Delta-5,23-stigmastadienol & $<0.10$ & $<0.10$ & $<0.10$ & $<0.10$ & $<0.10$ & $<0.10$ \\
\hline Clerosterol & 0.80 & 0.70 & 0.70 & 0.70 & 0.50 & 0.90 \\
\hline Beta-sitosterol & 58.70 & 56.30 & 83.40 & 58.40 & 44.20 & 56.70 \\
\hline Sitostanol & 0.70 & 0.70 & 1.40 & 0.70 & 0.40 & 1.00 \\
\hline Delta-5-avenasterol & 2.50 & 2.50 & 6.20 & 2.40 & 2.00 & 3.00 \\
\hline Delta-7,9(11)-stigmastadienol & 0.20 & $<0.10$ & $<0.10$ & $<0.10$ & $<0.10$ & $<0.10$ \\
\hline Delta-5,24-stigmastanediol & 0.40 & 0.20 & 0.80 & 0.80 & 0.20 & 0.80 \\
\hline Delta-7-stigmastenol & 0.40 & 0.90 & 0.70 & 0.60 & 0.20 & 1.00 \\
\hline Delta-7-avenasterol & 0.20 & 0.70 & 0.80 & 0.30 & 0.10 & 0.50 \\
\hline
\end{tabular}


Table 5. Organic acids (mg/kg) in cocoa mass (CM), cocoa 22-24 (C), hazelnut paste (HP), dark chocolate (D), milk chocolate (M), and gianduja chocolate (G) analysed at production time $(\mathrm{t} 0)$. Data represent the mean $\pm \mathrm{SD}(n=3)$. Within each row, different superscript letters indicate statistically different values among samples according to a post-hoc comparison (Tukey's test) at $p \leq 0.05$.

\begin{tabular}{|c|c|c|c|c|c|c|}
\hline Organic Acids & $\mathrm{CM}$ & $\mathrm{C}$ & HP & D & $\mathbf{M}$ & $\mathrm{G}$ \\
\hline Ossalic acid & $889 \pm 27.3^{b}$ & $1824 \pm 33.2^{a}$ & $195 \pm 4.6^{\mathrm{d}}$ & $421 \pm 9.7^{c}$ & $37 \pm 0.4^{\mathrm{f}}$ & $79 \pm 12.7^{\mathrm{e}}$ \\
\hline Citric acid & $1765 \pm 69.7^{b}$ & $3640 \pm 51.2^{a}$ & $547 \pm 36.1^{d}$ & $1163 \pm 98.5^{c}$ & $1681 \pm 78.5^{b}$ & $1253 \pm 34.7^{c}$ \\
\hline Acetic acid & $902 \pm 13.4^{b}$ & $2750 \pm 5.8^{\mathrm{a}}$ & $610 \pm 7.9^{c}$ & $109 \pm 0.3^{\mathrm{e}}$ & $547 \pm 34.6^{\mathrm{d}}$ & $454 \pm 78.2^{d}$ \\
\hline L-malic acid & $444 \pm 15.9^{b}$ & n.d. & $1524 \pm 167.4^{\mathrm{a}}$ & $399 \pm 2.5^{c}$ & $194 \pm 12.8^{d}$ & $1388 \pm 54.9^{a}$ \\
\hline Lactic acid & $570 \pm 3.3^{b}$ & n.d. & $17 \pm 0.7^{\mathrm{e}}$ & $194 \pm 0.8^{\mathrm{d}}$ & $874 \pm 56.8^{a}$ & $434 \pm 23.8^{c}$ \\
\hline Formic acid & n.d. & n.d. & $208 \pm 16.1^{b}$ & $843 \pm 6.4^{\mathrm{a}}$ & $229 \pm 34.1^{b}$ & $202 \pm 5.8^{b}$ \\
\hline Tartaric acid & $235 \pm 15.7$ & n.d. & n.d. & n.d. & n.d. & n.d. \\
\hline Succinic acid & $48 \pm 0.7^{b}$ & n.d. & n.d. & n.d. & $54 \pm 4.7^{\mathrm{a}}$ & n.d. \\
\hline
\end{tabular}

n.d. $=$ not detectable.

Table 6. Volatile compounds (mg/kg) in cocoa mass (CM), cocoa 22-24 (C), hazelnut paste (HP), dark chocolate (D), milk chocolate (M), and gianduja chocolate (G) analysed at production time $(\mathrm{t} 0)$. Data represent the mean $\pm \mathrm{SD}(n=3)$. Within each row, different superscript letters indicate statistically different values among samples according to a post-hoc comparison (Tukey's test) at $p \leq 0.05$.

\begin{tabular}{|c|c|c|c|c|c|c|c|c|}
\hline Volatile Compounds & $\mathrm{CM}$ & $\mathrm{C}$ & HP & D & $\mathbf{M}$ & G & References & Descriptors \\
\hline 2,5-Dimethylpyrazine & n.d. & $22.69 \pm 2.20^{\mathrm{a}}$ & $5.21 \pm 1.54^{b}$ & n.d. & $0.46 \pm 0.07^{\mathrm{d}}$ & $0.95 \pm 0.02^{c}$ & $0.23-1.69$ & cocoa, roast nuts \\
\hline 2,6-Dimethylpyrazine & $3.32 \pm 0.03^{b}$ & $2.36 \pm 0.47^{c}$ & $12.67 \pm 2.58^{\mathrm{a}}$ & $15.94 \pm 3.19^{\mathrm{a}}$ & n.d. & $14.10 \pm 1.19^{\mathrm{a}}$ & $0.11-0.39$ & nutty, coffee, green \\
\hline 2,3,5-Trimethylpyrazine & $41.75 \pm 7.39^{a}$ & $19.18 \pm 3.57^{b}$ & $3.63 \pm 0.45^{\mathrm{d}}$ & $7.38 \pm 2.69^{c}$ & $0.12 \pm 0.02^{\mathrm{f}}$ & $0.33 \pm 0.17^{\mathrm{e}}$ & $0.21-1.71$ & cocoa, roast nuts, peanut \\
\hline 2,3,5,6-Tetramethylpyrazine & $6.50 \pm 1.51^{\mathrm{a}}$ & $4.50 \pm 2.11 \mathrm{a}^{\mathrm{b}}$ & $2.03 \pm 1.26^{c}$ & $8.15 \pm 1.89^{a}$ & $1.58 \pm 0.11^{\mathrm{c}}$ & $4.53 \pm 0.57^{a, b}$ & $0.52-8.28$ & chocolate, cocoa, coffee \\
\hline Benzaldehyde & $0.59 \pm 0.31^{\mathrm{d}}$ & $3.86 \pm 0.12^{b}$ & $0.48 \pm 0.04^{\mathrm{e}}$ & $7.52 \pm 3.50^{\mathrm{a}}$ & $1.80 \pm 0.60^{c}$ & $0.73 \pm 0.29^{d}$ & $0.5-1.89$ & bitter \\
\hline 2-Acetyl-5-methylfuran & n.d. & $5.06 \pm 0.92^{\mathrm{a}}$ & $1.43 \pm 0.16^{\mathrm{b}}$ & $1.76 \pm 0.45^{\mathrm{b}}$ & $0.50 \pm 0.14^{c}$ & $0.43 \pm 0.01^{\mathrm{c}}$ & & \\
\hline 2-Phenylacetaldehyde & $0.55 \pm 0.07^{c}$ & $2.15 \pm 0.75^{b}$ & $4.02 \pm 0.35^{\mathrm{a}}$ & $1.47 \pm 1.12 b^{c}$ & $2.59 \pm 0.54^{b}$ & $1.55 \pm 0.07 b^{c}$ & $2-8.90$ & berry, nutty \\
\hline$\alpha$-Terpenilformato & $0.16 \pm 0.01^{c}$ & n.d. & $0.72 \pm 0.11^{b}$ & n.d. & $0.71 \pm 0.22^{b}$ & $2.89 \pm 0.34^{\mathrm{a}}$ & $0-0.38$ & herbaceous, citrus \\
\hline Benzyl acetate & $0.37 \pm 0.29 \mathrm{~d}$ & n.d. & $1.92 \pm 0.01^{\mathrm{a}}$ & $0.59 \pm 0.12^{c}$ & $0.45 \pm 0.04^{d}$ & $1.32 \pm 0.13^{b}$ & $0-0.033$ & floral, jasmine \\
\hline Octanoic acid & $0.40 \pm 0.29 \mathrm{~b}$ & $1.93 \pm 0.96^{\mathrm{a}}$ & $1.12 \pm 0.09^{\mathrm{a}}$ & $1.62 \pm 0.02^{\mathrm{a}}$ & $0.61 \pm 0.23 \mathrm{~b}$ & $0.85 \pm 0.01^{\mathrm{a}}$ & $0.021-0.37$ & unpleasant, oily, fatty \\
\hline 2-Acetyl pyrrole & $0.18 \pm 0.03^{c}$ & $2.74 \pm 1.71^{\mathrm{a}}$ & $1.52 \pm 0.31^{\mathrm{a}}$ & n.d. & $0.36 \pm 0.01^{b}$ & $1.58 \pm 1.11^{\mathrm{a}}$ & $0.021-0.38$ & bread, walnut, licorice \\
\hline 3-Hydroxy-2-methylpyridine & n.d. & $1.63 \pm 0.06^{b}$ & $1.84 \pm 0.32^{b}$ & $4.90 \pm 1.94^{\mathrm{a}}$ & $0.59 \pm 0.04^{d}$ & $0.94 \pm 0.06^{c}$ & $0.14-0.38$ & wizened \\
\hline 2,3-Dihydro-3,5-dihydro-6-methyl-4-pyrone & n.d. & n.d. & n.d. & n.d. & $0.50 \pm 0.14$ & n.d. & $0.28-1.87$ & roasted \\
\hline 3,5-Hydroxy-6-methyl-4-pyrone & n.d. & n.d. & n.d. & n.d. & $2.59 \pm 0.54$ & n.d. & $0.02-0.37$ & roasted \\
\hline
\end{tabular}

n.d. = not detectable. 


\subsubsection{Chocolates}

For D and M chocolates, the content of ash, proteins, and fats (Table 3) confirmed CREA [50] and other authors' findings [19]. The ash levels were similar between samples and minor at $2 \%$, while proteins were higher in the D sample (at 7.2\%) than in the M sample with a content of $6.4 \%$ (Table 3). Since D is obtained from the processing of cocoa mass (CM), its results (Table 4) outlined a composition of mainly oleic acid (C18:1), stearic acid (C18:0), palmitic acid (C16:0), and small quantities of linoleic acid (C18:2), confirming what has been reported in the literature [51,52]. As observed by Çakmak et al. [52], oleic acid (C18:1), stearic acid (C18:0), palmitic acid (C16:0), and linoleic acid (C18:2) prevailed in the cocoa mass $(\mathrm{CM})$.

The theobromine (Table 3) at a concentration of about $7.3 \mathrm{mg} / \mathrm{g}$ for D and almost $4 \mathrm{mg} / \mathrm{g}$ for $\mathrm{M}$ agreed with what has been described by Belščak-Cvitanović et al. [53]. Besides, the concentration in D was compliant with Meng et al. [54], who indicated a range of 237-519 mg of theobromine per $50 \mathrm{~g}$ portion of dark chocolate. The total soluble polyphenols, with a value of about $2.1 \mathrm{mg} \mathrm{GAE} / \mathrm{g}$, and caffeine, with more than $21 \mathrm{mg} / 100 \mathrm{~g}$ in D (Table 3), were similarly remarked upon in a previous study [53].

Citric, oxalic, acetic, malic, lactic, and formic acids were present in all the samples (Table 5), with formic acid in the D sample having a value of $843 \mathrm{ppm}$, which is greater than in other research $[10,55]$. The formic acid did not seem to come from the raw materials, but rather from the transformations that occurred during the process: despite its volatility, it was maintained at medium-high levels in the finished chocolates (Table 5). Tartaric acid was completely absent in the chocolates (Table 5), while succinic acid was only found in the M sample with almost a 55-ppm concentration, probably deriving from the added milk.

Finally, considering the volatile compounds detected in the D, M, and G samples, Table 6 shows that $\mathrm{D}$ chocolate was characterized by a higher level of pyrazines and benzaldehyde, reaching a value of about 31.5 and $7.5 \mathrm{ppm}$, respectively, in line with the results of other studies [48,56]. As a matter of fact, pyrazines contribute to $40 \%$ of the volatile compounds of roasted cocoa aroma with multiple descriptors. In the study of Liu et al. [48], dark chocolate was characterized by a cocoa flavor with malty, nutty, and toasted notes. Furthermore, Aprotosoaie et al. [57] stated that different cocoa types may exhibit various and specific flavors since the concentration and sensory characters of these compounds vary significantly, depending on the nature and origin of the identified molecules. The literature has stressed a possible microbial fermentation derivation of Bacillus subtilis and Bacillus megatrium; however, most cocoa and chocolate pyrazines originate in Strecker degradation and Maillard reactions, and need heat and precursors such as aminocinetones, acetoin, and diacetyl [56]. This allows us to conclude that the roasting of both cocoa and hazelnuts has a considerable impact on the aroma profile of the chocolates and related ingredients.

\subsubsection{Ingredients}

Polyphenols exceeded $0.30 \mathrm{mg}$ GAE/g (Table 3) in HP, as also reported in other research [58,59]. The highest concentrations were found in the $C$ sample, with more than $7 \mathrm{mg} \mathrm{GAE} / \mathrm{g}$ of polyphenols, as in Belšcak et al. [27], although the literature mentions very variable polyphenol contents, depending on the geographical origin of the beans, degree of maturation, processing, and packaging $[7,9,11,29,46]$. The content in theobromine and caffeine of the $C$ sample under study (Table 2) was coherent with what was reported by Jalil and Ismail [60], whilst the $\mathrm{pH}$ value (Table 3) matched Miller et al.'s results [61], since this sample may be considered as quite alkalized cocoa, showing a $\mathrm{pH}$ value greater than 8.00. The alkalization of cocoa powder causes a decrease in the total polyphenols and an increase in $\mathrm{pH}$ up to 8.0. Natural cocoa powder has a $\mathrm{pH}$ of about 5.3-5.8, while alkalized cocoa powder may be classified according to treatment: light treatment ( $\mathrm{pH}$ 6.5-7.2), medium treatment ( $\mathrm{pH} 7.2-7.6)$, and heavy treatment ( $\mathrm{pH} 7.6$ and above) [61].

The overall fat content (Table 3) was lower than what was reported by CREA [50], with the most in HP and the least in the $\mathrm{C}$ sample. The lipid content is influenced by the geographical origin 
of cocoa, ranging from $16 \%$ to $22 \%$ for commercial typologies [6]. The fatty acid composition of $C$ (Table 4) matched what was stated by Elkhori et al. [51]. As for the triglycerides of cocoa butter, our results (Table 4) outlined mainly oleic acid (C18:1), stearic acid (C18:0), palmitic acid (C16:0), and small amounts of linoleic acid (C18:2), as presented in the literature [51], while the Vitamin E content found in C (Table 3), i.e., more than 9\%, complied with Lipp et al.'s findings [62]. The saturated, monounsaturated, and polyunsaturated fatty acid content (Table 4) of CM agreed with the values presented by Çakmak et al. [52]. Oleic acid exceeded 30\% in all of the samples, reaching almost $83 \%$ in the HP sample (Table 3). Moreover, there were other fatty acids (lauric, myristic, myristoleic, pentadecanoic, pentadecenoic, palmitoleic, heptadecanoic, heptadecenoic, linolenic, arachic, eicosadienoic and eicosatrienoic, behenic, and lignoceric) ranging between $0.01 \%$ and $1 \%$ (Table 5), as found by Bignami et al. [63]. Finally, vitamin E (Table 2) ranged around 300 ppm, confirming what was previously reported [63].

Despite various works being carried out on the lipid fraction of hazelnuts, few data are currently available on the organic acids of HP [63-65], which, in our study (Table 5), showed malic as the most abundant acid. Citric, oxalic, and acetic were the only acids detected in the $\mathrm{C}$ sample (Table 5). Tartaric and succinic acid were observed in the CM (Table 5), but they were completely absent in the other samples, while formic acid was only present in the HP (Table 4). Succinic acid contained in CM at a concentration of $48 \mathrm{ppm}$ was probably a derived fermentation product. D-L lactic and L-malic acid were present in both CM and HP (Table 5).

The analysis of the aroma compounds led to the identification of the only octanoic acid as off-flavor, which showed the highest amounts in C (Table 6). Among the main contributors to the overall profile of cocoa producing, the greatest impacts to chocolate aroma $[48,56]$, tetramethylpyrazine and trimethylpyrazine, responsible for milk coffee-mocha roasted, nutty, and earthy aromas, respectively, were present, especially in CM (Table 5), whilst phenylacetaldehyde (rosy-like aroma) showed the highest value in HP (Table 5).

\subsection{Eighteen-Month Evolution of Chocolates and Ingredients: Nutritional and Sensory Changes}

As chocolate is a continuous lipid phase, the structural changes in its fat matter may alter volatile release, thus changing the flavor profile of the chocolate $[11,14,17,47,48,56,57,66]$. As a consequence, chocolates and the related ingredients under study were chemically checked during the 18 months of storage for peroxide and acidity values, polyphenols, and vitamin E [67]. This chemical-physical monitoring was applied with an eighteen-weekly check frequency and outlined for both chocolates and ingredients, and the results are reported in Figure 1.

On the other hand, the sensory analysis was performed at $\mathrm{t} 0, \mathrm{t} 1, \mathrm{t} 2, \mathrm{t} 3$, and $\mathrm{t} 4$ by means of QDA profiling that allowed identifying significant trends of increase or decrease in the visual, auditory, mechanical, and flavor perceptions of the samples $[11,68]$. In the QDA sensory sheet, panelists could indicate descriptors related to the sensory flaws: this was useful for displaying any "anomalies" that arose during storage. The evolution of the sensory attributes of each sample during the eighteen months of conservation is represented in Figures 2 and 3 for ingredients and chocolates, respectively. 

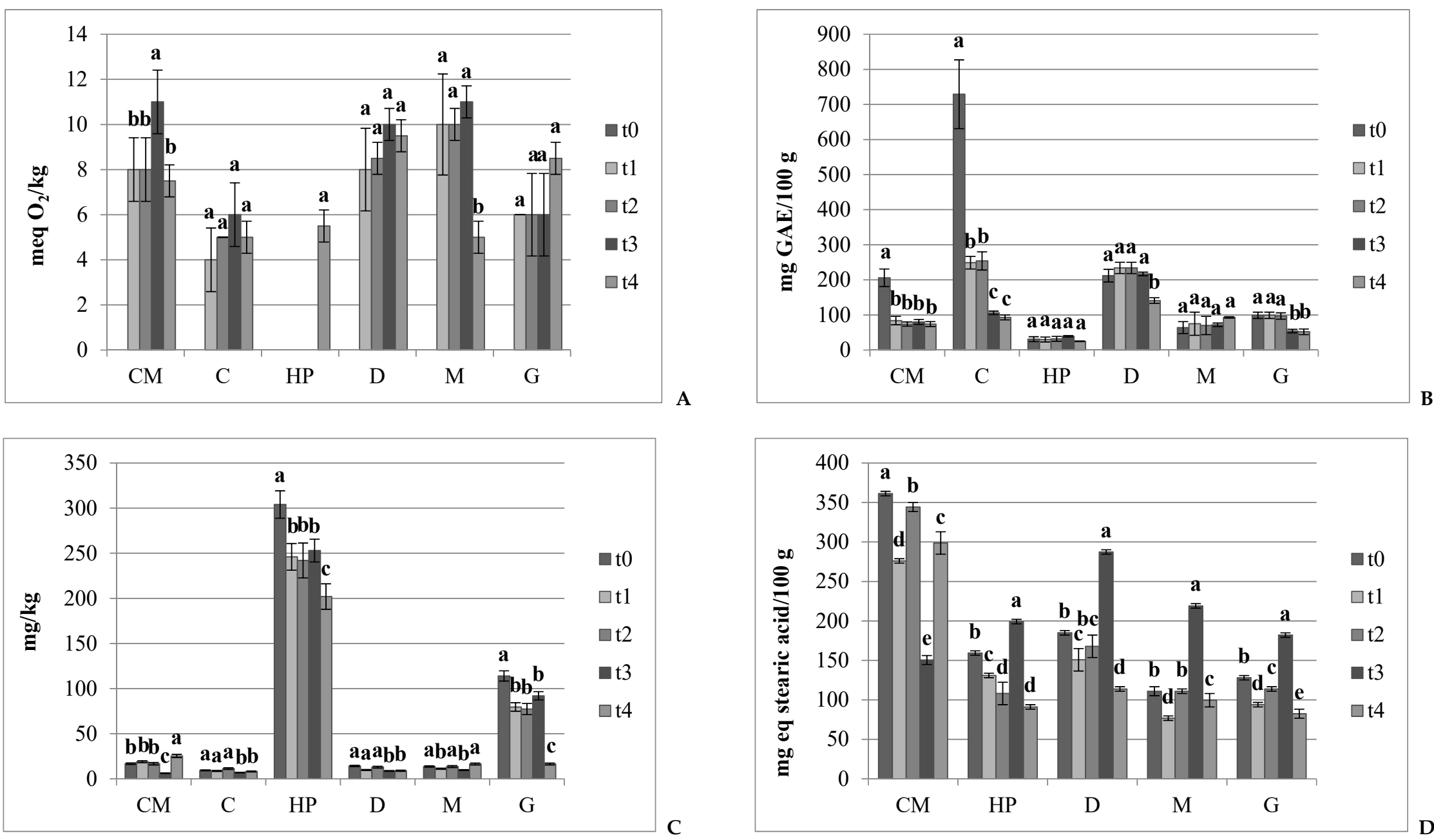

Figure 1. Evolution of the lipid and phenolic matrix at $\mathrm{t} 0, \mathrm{t} 1, \mathrm{t} 2, \mathrm{t} 3$, and $\mathrm{t} 4$ of (A) Peroxide values (expressed as meq of $\mathrm{O}_{2} / \mathrm{kg}$ ); (B) Unoxidized polyphenol concentration (expressed as mg of GAE per $100 \mathrm{~g}$ ); (C) Vitamin E concentration (mg/kg); (D) Acidity values (expressed as mg of stearic acid equivalents per $100 \mathrm{~g}$ ). Data represent the mean \pm SD $(n=3)$. Within each sample, different letters indicate statistically different values among times according to a post-hoc comparison (Tukey's test) at $p \leq 0.05$. 


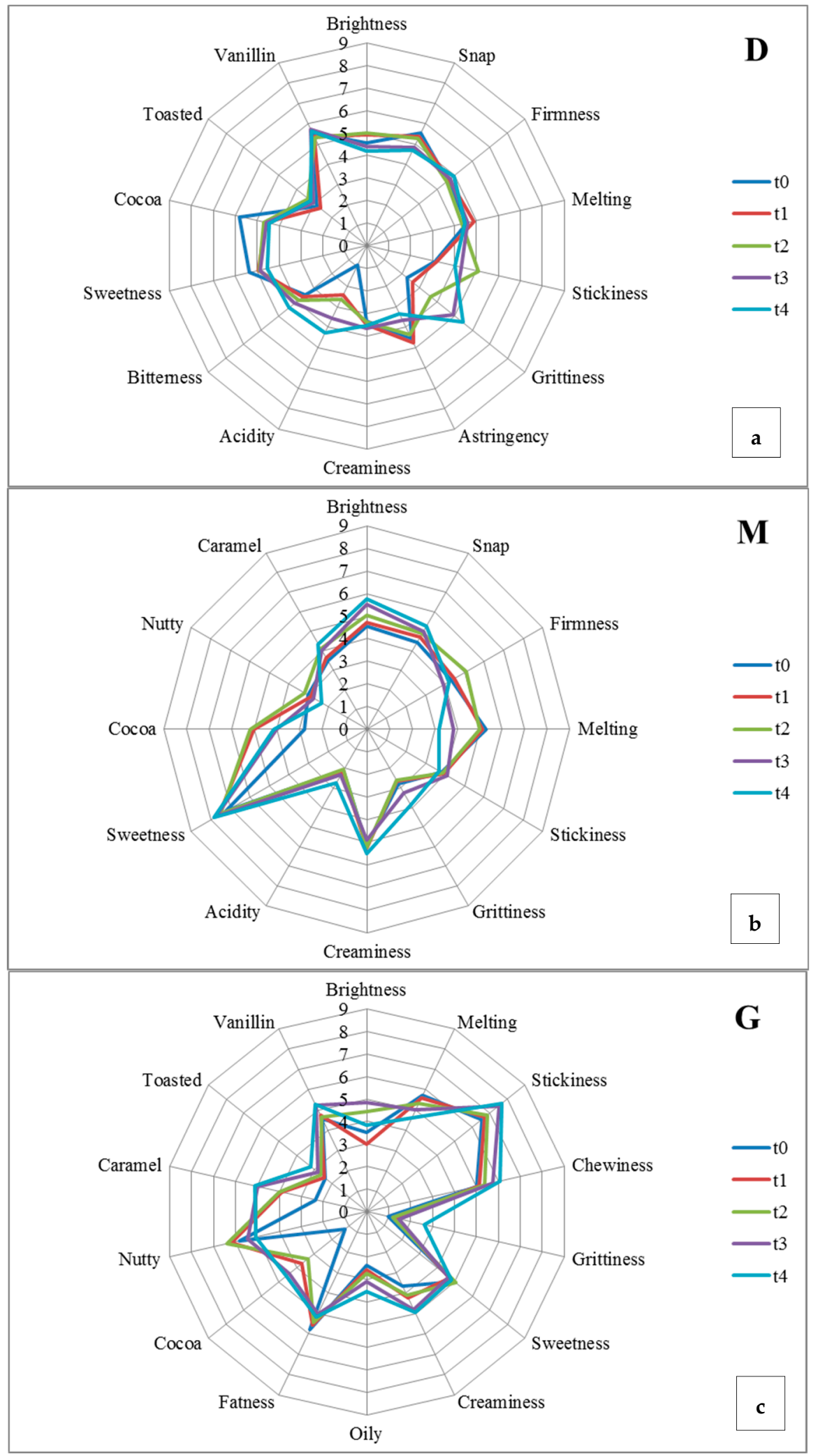

Figure 2. Sensory profile at $\mathrm{t} 0, \mathrm{t} 1, \mathrm{t} 2, \mathrm{t} 3$, and $\mathrm{t} 4$ of the dark chocolate (D, subfigure a), milk chocolate (M, subfigure b), and gianduja chocolate ( $G$, subfigure $\mathbf{c})$. 


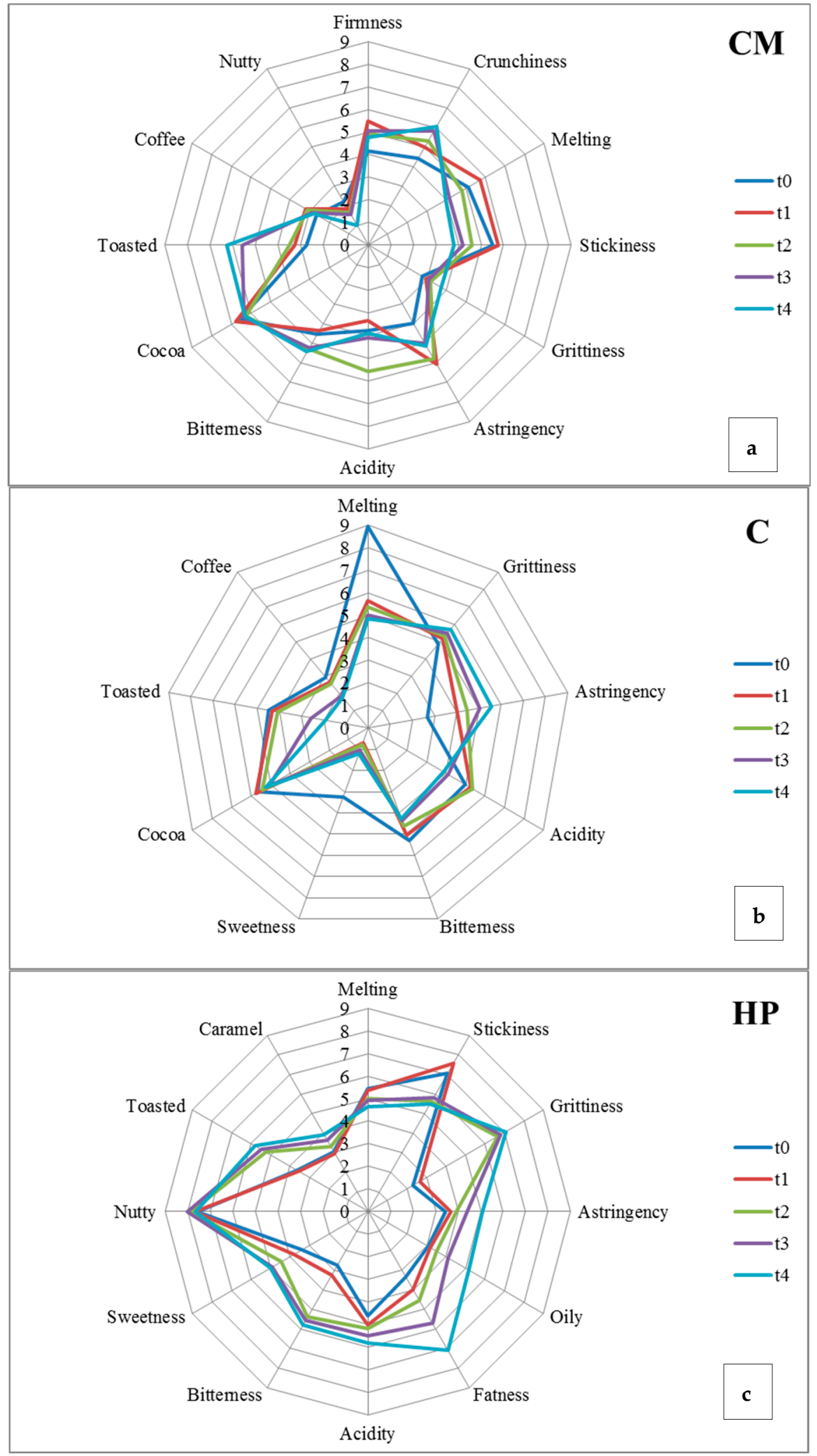

Figure 3. Sensory profile at $\mathrm{t} 0, \mathrm{t} 1, \mathrm{t} 2, \mathrm{t} 3$, and $\mathrm{t} 4$ of the cocoa mass (CM, subfigure a), cocoa 22-24 (C, subfigure $\mathbf{b})$, and hazelnut paste (HP, subfigure $\mathbf{c})$. 


\subsubsection{Chemo-Sensory Evolution of Chocolates}

In the milk chocolate (M sample), brightness and snap increased over time (Figure 2), whereas the intensity of brightness remained constant in $\mathrm{D}$, where the snap became less intense from $\mathrm{t} 0$ to $\mathrm{t} 4$ (Figure 2a). This was in accordance with Machálková et al. [66], who found a slight deterioration of some mechanical descriptors in the chocolate samples stored at $20^{\circ} \mathrm{C}$. The firmness did not change significantly in D and M samples (Figure 2a,b), while the melting dropped in $\mathrm{G}$ and $\mathrm{M}$ chocolate (Figure 2c,b). In this regard, Thamke et al. [68] concluded that chocolate with a lower cocoa content was characterized by the greatest melting and creaminess, while the product with the highest cocoa content was characterized as dry dough. This was confirmed by the results from Figure $2 c$, which show a lower value of creaminess in D chocolate than in M and G samples.

It is interesting to compare the behavior of fat matter with the modification in structural properties observed in chocolate samples and previously described, since the physical state of triglycerides is known to affect the firmness, creaminess, and melting of a chocolate-based product [67]. The alteration of the physical state of fat matter, which is widely observed over time in the storage of fatty food products, is mainly due to rancidity phenomena starting with the hydrolysis of triglycerides giving rise to free fatty acids, which in turn undergo oxidation [69]. This emphasized the role acquired by the monitoring of both acidity and peroxide values that, together with sensory outcomes, in terms of perceived acidity, may help to get quick and consistent information about the fat matter evolution of chocolate-based products during their storage.

Our results evidenced a reduction in the acidity in the first eighteen weeks (t1) (Figure 1D), followed by a fast and similar increase in all the samples after $t 2$ with values of almost 290, 220, and $185 \mathrm{mg}$ eq. stearic acid/100 g in D, M, and G samples, respectively. Afterwards, it fell again until the eighteenth month of storage ( $t 4)$. Considering the $\mathrm{M}$ chocolate (Figure $2 b$ ), acidity increased, this was only slightly with respect to the D sample (Figure 2a). Regarding peroxides, the three chocolates maintained a value ranging between 5 and 11 meq of $\mathrm{O}_{2} / \mathrm{kg}$ (Figure $1 \mathrm{~A}$ ). In particular, the results showed no detectable peroxides at the production time $(\mathrm{t} 0)$, while at the $\mathrm{t} 1$, there was a significant increase, especially in $M$ and $D$ chocolate. The fact that in $G$, the peroxide value remained lower than the other two chocolates, confirms the observations of other authors [70,71], who have attributed this behavior to the phytochemical compounds present in dried fruit, in this case, hazelnuts. As observed for $\mathrm{M}$ samples after 18 months (Figure 1A), the peroxide value decreases due to them changing into short-chain aldehydes, or in secondary products deriving from their decomposition [69,72]. Therefore, despite chocolates having a high fat content (Table 3), the lipid oxidation at the end of storage (Figure 1A) was very slow. Both the lyophobic and the lyophilic antioxidants were supposed to perform a continuously protective activity towards fats $[14,69]$ due to their well-known biological effects $[3,16,17,27,53,60,61,73,74]$.

At the end of the eighteen months, the residual content in polyphenols of the chocolates ranged from 50 to $217 \mathrm{mg}$ of GAE per $100 \mathrm{~g}$, highlighting a significant difference between the different types of chocolate. Phenolic substances are involved in the chocolate's flavor and in the primary sensory characteristics as bitterness and astringency. The analysis (Figure 1B) showed a slight increase in D and $M$ from time $t 0$ to time $t 1$, while in $G$, the concentration remained constant until $t 2$, and then decreased significantly up to the end of storage (t4), reaching the value of $54 \mathrm{mg} \mathrm{GAE} / 100 \mathrm{~g}$ (Figure 1B). Although D chocolate showed an important loss of polyphenols (Figure 1B), it maintained the highest content at the end of storage ( $\mathrm{t} 4)$ when the astringency was perceived to be at a medium level, contrary to $\mathrm{M}$ and G chocolate (Figure 2b,c). Studies [11-13,66,67] have already observed the depletion of polyphenols in cocoa-based products during storage, correlating this loss with their oxidation in the corresponding quinones, which might lead to increases in bitterness, as outlined in D chocolate (Figure 2a). In the same sample, starting from $t 2$, some panelists marked descriptors related to oxidation as "pungent", "closed", "cork", and "dried fig". This was in line with Subramaniam [72], who reported that dark chocolate loses some of its chocolate flavor and develops a "stale note" over time. Additionally, she 
reported that the chocolate develops a stale, "cardboardy" flavor due to oxidative rancidity prior to the onset of fat bloom.

In this regard, results from vitamin E showing a protective antioxidant effect with a reduction potential of $500 \mathrm{mV}$, comparable to that of epigallocatechin gallate $(430 \mathrm{mV})$ [75], were particularly interesting in the sample derived from hazelnut, i.e., in $\mathrm{G}$ chocolate, where a moderate decrease was recorded at $\mathrm{t} 1$ and $\mathrm{t} 4 \mathrm{control}$ times (Figure $1 \mathrm{C}$ ). The concentration in vitamin $\mathrm{E}$ detected in $\mathrm{G}$ supported its non-significant lipid oxidation (Figure 1A), even if the G polyphenol content was lower than in D and $\mathrm{M}$ chocolates (Figure 1B). The G sample (Figure 2c) showed a growth in stickiness, chewiness, creaminess, and grittiness. A drop in the intensity of positive perceptions was also reported by Bomba [76], who noted that the addition of nuts to chocolate shortens its shelf-life, even though the antioxidants in chocolate may be of some benefit to the oil in the nuts. In this regard, the oily attribute continuously rises, even if the fatness perception slightly decreases (Figure 2c). In the M chocolate (Figure 2b), the attributes of "liquorice" and "aged" have also been reported, although at medium levels. Additionally, milk chocolate flavors tend to blend with aging, but extended aging may result in undesirable fruity notes [77]. Liu et al. [48] reported that the typical flavors of milk chocolate are milky, nutty, and caramel with coconut notes, as observed in the present study (Table 5).

\subsubsection{Chemo-Sensory Evolution of Ingredients}

As regards the evolution of ingredients, the three semi-finished products maintained a peroxide value (Figure 1A) corresponding to a good state of conservation [46], ranging between 4 and 11 meq of $\mathrm{O}_{2} / \mathrm{kg}$, and even if from $\mathrm{t} 2$, the parameter was significantly elevated in the CM. On the contrary, in the HP, the data is not detectable in the first three control times and only at $t 4$ was a value of almost 6 meq of $\mathrm{O}_{2} / \mathrm{kg}$ registered (Figure 1A). This result confirmed the observations of other authors [70], who attributed this behavior to the phytochemical compounds present in the hazelnuts. Indeed, in vitro studies have shown that incubating cells with nut extracts very rich in polyphenols can inhibit oxidative susceptibility [64]. It should be noted that, as observed for $C$ and $C M$, after 18 months (Figure 1A), the peroxide value may decrease when the hydro-peroxides formed evolve into short-chain aldehydes, i.e., in secondary products deriving from their decomposition. Conversely, the acidity was risen in a linear way only in the HP sample (Figure 3c), while in $\mathrm{CM}$ and $\mathrm{C}$, the trend of this attribute was quite fluctuating (Figure 3a,b). In detail, the acidity of CM and HP decreased at $\mathrm{t} 1$, whereas $\mathrm{C}$ had undetectable values at each sampling point (Figure 1D). After the $t 2$ control, the acidity of $C M$ increased, while in HP, it continued to decrease, as also reported by Fardelli [70], who analyzed roasted and natural nuts for nine months of storage.

As well as in chocolates and other studies [12-15,76], a decrease in the total polyphenol content due to their oxidation was especially observed in $\mathrm{CM}$ and $\mathrm{C}$ from time t0 to time $\mathrm{t} 1$ (Figure 1B) and then from the $\mathrm{t} 2$ to $\mathrm{t} 3$ for the $\mathrm{C}$ sample till the lowest value of $93 \mathrm{mg} \mathrm{GAE} / 100 \mathrm{~g}$. On the other hand, the unoxidized polyphenols remained almost constant for HP throughout the full storage period, ranging between 25 and $39 \mathrm{mg} \mathrm{GAE} / 100 \mathrm{~g}$ (Figure 1B). This was quite different from what was observed by Fardelli [70], who reported decreases in polyphenols during nine-month storage of natural and roasted nuts.

The results of vitamin $\mathrm{E}$ indicated no significant change during the whole storage time in the HP under examination (Figure 1C), with the exception of a decrease at times $\mathrm{t} 1$ and $\mathrm{t} 4$. The retained level of vitamin E explains why the HP did not demonstrate detectable lipid oxidation (Figure 1A), even though it had polyphenol content levels that were lower than other samples (Figure 1B). The antioxidant properties of tocopherols come from their ability to donate their phenolic hydrogen to lipid free radicals and to retard the autocatalytic lipid peroxidation processes [78]. Moreover, they show a good stability: after six months of maintenance at room temperature, a 13\% decrease in antioxidant activity was already observed [79].

Finally, regarding flavors, an emphasis should be placed on the toasted aroma, whose intensity increased during storage for $\mathrm{CM}$ and $\mathrm{HP}$, while it decreased drastically for C. During the eighteen 
months of storage, in the $\mathrm{CM}$ (Figure 3a), the nutty aroma decreased, while cocoa and coffee remained stable. In $\mathrm{C}$, the coffee aroma dropped significantly in the last months of storage, while the cocoa intensity remained constant (Figure $3 b$ ). On the contrary, in HP, the caramel aroma increased, in addition to oily and fattiness perception (Figure 3c).

\section{Conclusions}

In the present work, two main groups of cocoa-based foods (chocolates and related ingredients) were investigated by monitoring the evolution of some nutritional components during eighteen months of storage under conditions simulating a point of sale or factory warehouse.

Although the matrices under study contained variable amounts of cocoa butter and hazelnut oil, the evolution of their fats revealed only slow oxidation phenomena. Due to the known effects of catechins, flavonols, and proanthocyanins against lipid peroxidation, the polyphenols measured in chocolates and related ingredients continued to exhibit their protective activity towards fats, even though a general loss in unoxidized polyphenols was observed over time. Finally, despite some alteration of mechanical and structural properties, as well as some losses in aroma, the samples exhibited good sensory scores for the descriptive analysis till the eighteenth month of storage.

Since chocolate, as part of a balanced diet, is becoming a commodity with health and nutritional benefits, these outcomes are really useful for confectionery companies in order to gain detailed information about the state of chocolates and related ingredients which is perceptible by humans and associated with their nutritional composition. Enhancing this knowledge represents a stimulus to people involved in this kind of production, processing, and consumption.

Author Contributions: Conceptualization and methodology, M.L.; Writing—review and editing, A.R.

Funding: This research was funded by the Venchi S.p.A. through grant from the Ministry of Economic Development for the "Industrial innovation project: New Technologies for the Made in Italy" (D.M. 22 December 2010; G.U.R.I. no. 53 of 5 March 2011).

Acknowledgments: The authors are grateful to Massimiliano Cavicchioli for technical support.

Conflicts of Interest: The authors declare no conflict of interest.

\section{References}

1. Lin, X.; Zhang, I.; Li, A.; Manson, J.E.; Sesso, H.D.; Wang, L.; Liu, S. Cocoa flavanol intake and biomarkers for cardiometabolic health: A systematic review and meta-analysis of randomized controlled trials. J. Nutr. 2016, 146, 2325-2333. [CrossRef]

2. Ludovici, V.; Barthelmes, J.; Nägele, M.P.; Enseleit, F.; Ferri, C.; Flammer, A.J.; Ruschitzka, F.; Sudano, I. Cocoa, blood pressure, and vascular function. Front. Nutr. 2017, 4, 36. [CrossRef]

3. Kim, J.; Kim, J.; Shim, J.; Lee, C.Y.; Lee, K.W.; Lee, H.J. Cocoa phytochemicals: Recent advances in molecular mechanisms on health. Crit. Rev. Food Sci. Nutr. 2014, 54, 1458-1472. [CrossRef]

4. Fung, T. Healthy Eating: A Guide to the New Nutrition; Harvard School of Public Health, Nutrition Department: Boston, MA, USA, 2011; p. 48.

5. Manach, C.; Scalbert, A.; Morand, C.; Remesy, C.; Jimenez, L. Polyphenols: Food sources and bioavailability. Am. J. Clin. Nutr. 2004, 79, 727-747. [CrossRef]

6. Balestieri, F.; Marini, D. Metodi di Analisi Chimica dei Prodotti Alimentari; Monolite: Roma, Italy, 1996; ISBN 8873310052.

7. Badrie, N.; Bekele, F.; Sikora, E.; Sikora, M. Cocoa agronomy, quality, nutritional, and health aspects. Crit. Rev. Food Sci. Nutr. 2015, 55, 620-659. [CrossRef]

8. EFSA Panel on Dietetic Products, Nutrition and Allergies (NDA). Scientific Opinion on the substantiation of a health claim related to cocoa flavanols and maintenance of normal endothelium-dependent vasodilation pursuant to Article 13(5) of Regulation (EC) No 1924/2006. EFSA J. 2012, 10, 2809.

9. Afoakwa, E.O. Chocolate Science and Technology; Wiley-Blackwell: Singapore, 2010.

10. Januszewska, R.; Viaene, J. Acceptance of chocolate by preference cluster mapping across Belgium and Poland. J. Euromark. 2002, 11, 61-86. [CrossRef] 
11. Popov-Raljić, J.V.; Laličić-Petronijević, J.G. Sensory properties and color measurements of dietary chocolates with different compositions during storage for up to 360 days. Sensors 2009, 9, 1996-2016. [CrossRef]

12. Kumara, B.; Jinap, S.; Che Man, Y.B.; Yusoff, M.S.A. Comparison of colour techniques to measure chocolate fat bloom. Food Sci. Technol. Int. 2003, 9, 295-299. [CrossRef]

13. Pastor, C.; Santamaria, J.; Chiralt, A.; Aguilera, J.M. Gloss and colour of dark chocolate during storage. Food Sci. Technol. Int. 2007, 13, 27-30. [CrossRef]

14. Simic, M.G.; Jovanovic, S.V.; Niki, E. Mechanisms of lipid oxidative processes and their inhibition. In Lipid Oxidation in Food; St. Angelo, A.J., Ed.; American Chemical Society: New York, NY, USA, 1992; pp. 14-32.

15. Jolic, S.M.; Redovnikovic, I.R.; Markovic, K.; Sipusic, D.I.; Delonga, K. Changes of phenolic compounds and antioxidant capacity in cocoa beans processing. Int. J. Food Sci. Technol. 2011, 46, 1793-1800. [CrossRef]

16. McShea, A.; Ramiro-Puig, E.; Munro, S.B.; Casadesus, G.; Castell, M.; Smith, M.A. Clinical benefit and preservation of flavonols in dark chocolate manufacturing. Nutr. Rev. 2008, 66, 630-641. [CrossRef]

17. Wollgast, J.; Anklam, A. Review on polyphenols in Theobroma cacao: Changes in composition during the manufacture of chocolate and methodology for identification and quantification. Food Res. Int. 2000, 33, 423-447. [CrossRef]

18. Commission Regulation (EC) No 2073/2005 of 15 November 2005 on microbiological criteria for foodstuffs. Off. J. Eur. Union 2005, OJL 338, 1-26.

19. ISO. ISO 4833-1:2013. Microbiology of Food and Animal Feeding Stuffs—Horizontal Method for the Enumeration of Microorganisms-Colony-Count Technique at $30{ }^{\circ} \mathrm{C}$; ISO: Geneva, Switzerland, 2013.

20. ISO. ISO 21528-1:2017. Microbiology of Food and Animal Feeding Stuffs-Horizontal Methods for the Detection and Enumeration of Enterobacteriaceae-Part 1: Detection and Enumeration by MPN Technique with Pre-Enrichment; ISO: Geneva, Switzerland, 2017.

21. ISO. ISO 4832:2006. Microbiology of Food and Animal Feeding Stuffs—Horizontal Method for the Enumeration of Coliforms-Colony-Count Technique; ISO: Geneva, Switzerland, 2006.

22. AOAC International. AOAC International. AOAC 931.04, Moisture in Cocoa Products. In Official Methods of Analysis of AOAC International, 17th ed.; AOAC International: Gaithersburg, MD, USA, 2013.

23. AOAC International. Total dietary fiber in foods, enzymatic-gravimetric method. In Official Methods of Analysis of AOAC International, 17th ed.; AOAC International: Gaithersburg, MD, USA, 2003.

24. Office International du Cacao et du Chocolat et de la Confiserie (OICCC). Analytical Method; OICCC: Brussels, Belgium, 1972.

25. AOAC Authors. Official Methods of Analysis Lipids, Fats and Oils Analysis Total Carotenoids_Item 22, 17th ed.; Association of Analytical Communities: Gaithersburg, MD, USA, 2006.

26. Calvo, P.; Castaño, Á.L.; Hernández, M.T.; González-Gómez, D. Effects of microcapsule constitution on the quality of microencapsulated walnut oil. Eur. J. Lipid Sci. Technol. 2011, 113, 1273-1280. [CrossRef]

27. Belšcak, A.; Komes, D.; Horzic, D.; Ganic, K.K.; Karlovic, D. Comparative study of commercially available cocoa products in terms of their bioactive composition. Food Res. Int. 2009, 42, 707-716. [CrossRef]

28. AOAC International. AOAC 939.02-1939, Protein (milk) in milk chocolate. Kjeldahl method. In Official Methods of Analysis of AOAC International, 17th ed.; AOAC International: Gaithersburg, MD, USA, 2000.

29. Adamson, G.E.; Lazarus, A.; Mitchell, A.E.; Prior, R.L.; Cao, G.; Jacobs, P.H.; Kremers, B.G.; Hammerstone, J.F.; Rucker, R.B.; Ritter, K.A.; et al. HPLC method for the quantification of procyanidins in cocoa and chocolate samples and correlation to total antioxidant capacity. J. Agric. Food Chem. 1999, 47, 4184-4188. [CrossRef]

30. López-Martìnez, L.; López-de-Alba, P.L.; Garcìa-Campos, R.; De León-Rodrìguez, L.M. Simultaneous determination of methylxanthines in coffees and teas by UV-Vis spectrophotometry and partial least squares. Anal. Chim. Acta 2003, 493, 83-94. [CrossRef]

31. Hečimović, I.; Belščak-Cvitanović, A.; Horžić, D.; Komes, D. Comparative study of polyphenols and caffeine in different coffee varieties affected by the degree of roasting. Food Chem. 2011, 129, 991-1000. [CrossRef]

32. EC Commission Regulation no. 2568/91, annex III, on the characteristics of olive oil and olive-residue oil and on the relevant methods of analysis. Off. J. Eur. Union 1991, OJL 248, 1.

33. Bonvehì, J.S. Investigation of aromatic compounds in roasted cocoa powder. Eur. Food Res. Technol. 2005, 221, 19-29. [CrossRef]

34. Murray, J.M.; Delahunty, C.M.; Baxter, I.A. Descriptive Sensory Analysis: Past, Present, and Future. Food Res. Int. 2001, 34, 461-471. [CrossRef] 
35. Stone, H.; Sidel, J. Sensory Evaluation Practices. In Food and Science Technology Series, 2nd ed.; Taylor, S., Ed.; Academic Press: Cambridge, MA, USA, 1992; ISBN 9780323139762.

36. ISO. ISO 8589:2007. Sensory Analysis—General Guidance for the Design of Test Rooms; ISO: Geneva, Switzerland, 2007.

37. ISO. ISO 5492:2008. Sensory Analysis-Vocabulary; ISO: Geneva, Switzerland, 2008.

38. ISO. ISO 8586:2012. Sensory Analysis_General Guidelines for the Selection, Training and Monitoring of Selected Assessors And Expert Sensory Assessors; ISO: Geneva, Switzerland, 2012.

39. Donadini, G.; Fumi, M.D.; Lambri, M. The hedonic response to chocolate and beverage pairing: A preliminary study. Food Res. Int. 2012, 48, 703-711. [CrossRef]

40. MacFie, H.J.; Bratchell, N.; Greenhoff, K.; Vallis, L.V. Designs to balance the effect of order of presentation and first-order carry-over effect in halls tests. J. Sens. Stud. 1989, 4, 129-148. [CrossRef]

41. Kemp, S.E.; Hollowood, T.; Hort, J. Sensory Evaluation: A Practical Handbook; Wiley-Blackwell: Singapore, 2009. [CrossRef]

42. Meilgaard, M.; Civille, G.V.; Carr, B.T. Sensory Evaluation Techniques, 4th ed.; CRC Press: Boca Raton, FL, USA, 2007.

43. Civille, G.V.; Lyon, B.G. Aroma and Flavor Lexicon for Sensory Evaluation: Terms, Definitions, References, and Examples; ASTM Data Series Publication DS 66; American Society for Testing and Materials (ASTM) International: West Conshohocken, PA, USA, 1996.

44. Thompson, J.L.; Drake, M.A.; Lopetcharat, K.; Yates, M.D. Preference mapping of commercial chocolate milks. J. Food Sci. 2004, 69, S406-S413. [CrossRef]

45. Ferreira, J.M.M.; Marcacini Azevedo, B.; Luccas, V.; Bolini, H.M.A. Sensory profile and consumer acceptability of prebiotic white chocolate with sucrose substitutes and the addition of goji berry (Lycium barbarum). J. Food Sci. 2017, 82, 818-824. [CrossRef]

46. Leite, P.B.; Da Silva Bispo, E.; Randomille De Santana, L.R. Sensory profiles of chocolates produced from cocoa cultivars resistant to Moniliophtora Perniciosa. Rev. Bras. Frutic. 2013, 35, 594-602. [CrossRef]

47. Gills, L.A.; Resurreccion, A.V.A. Sensory and physical properties of peanut butter treated with palm oil and hydrogenated vegetable oil to prevent oil separation. J. Food Sci. 2000, 65, 173-180. [CrossRef]

48. Liu, J.; Liu, M.; He, C.; Song, H.; Guo, J.; Wang, Y.; Yang, H.; Su, X. A comparative study of aroma-active compounds between dark and milk chocolate: Relationship to sensory perception. J. Sci. Food Agric. 2015, 95, 1362-1372. [CrossRef]

49. Marvig, C.L.; Kristiansen, R.M.; Madsen, M.G.; Nielsen, D.S. Identification and characterization of organisms associated with chocolate pralines and sugar syrups used for their production. Int. J. Food Microbiol. 2014, 185, 167-176. [CrossRef]

50. CREA (Il Centro di Ricerca per gli Alimenti e la Nutrizione). Available online: https://www.crea.gov.it/web/ alimenti-e-nutrizione/banche-dati/ (accessed on 31 October 2019).

51. Elkhori, S.; Parè, J.R.J.; Bélanger, J.M.R.; Pérez, E. The microwave-assisted process (MAPTM1): Extraction and determination of fat from cocoa powder and cocoa nibs. J. Food Eng. 2007, 79, 1110-1114. [CrossRef]

52. Çakmak, Y.S.; Güler, G.O.; Aktümsek, A. Trans fatty acid contents in chocolates and chocolate wafers in Turkey. Czech J. Food Sci. 2010, 28, 177-184. [CrossRef]

53. Belščak-Cvitanović, A.; Durgo, K.; Gačina, T.; Horžić, D.; Franekić, J.; Komes, D. Comparative study of cytotoxic and cytoprotective activities of cocoa products affected by their cocoa solids content and bioactive composition. Eur. Food Res. Technol. 2012, 234, 173-186. [CrossRef]

54. Meng, C.C.; Jalil, A.M.; Ismail, A. Phenolic and theobromine contents of commercial dark, milk and white chocolates on the Malaysian market. Molecules 2009, 14, 200-209. [CrossRef] [PubMed]

55. Copetti, M.V.; Iamanaka, B.T.; Mororó, R.C.; Pereira, J.L.; Frisvad, J.C.; Taniwaki, M.H. The effect of cocoa fermentation and weak organic acids on growth and ochratoxin A production by Aspergillus species. Int. J. Food Microbiol. 2012, 155, 158-164. [CrossRef] [PubMed]

56. Afoakwa, E.O.; Paterson, A.; Fowler, M.; Ryan, A. Flavor formation and character in cocoa and chocolate: A critical Review. Crit. Rev. Food Sci. Nutr. 2008, 48, 840-857. [CrossRef] [PubMed]

57. Aprotosoaie, A.C.; Luca, S.V.; Miron, A. Flavor chemistry of cocoa and cocoa products-An Overview. Compr. Rev. Food Sci. Food Saf. 2016, 15, 73-91. [CrossRef]

58. Abe, L.T.; Lajolo, F.M.; Genovese, M.I. Comparison of phenol content and antioxidant capacity of nuts. Ciênc. Tecnol. Aliment. 2010, 30, 254-259. [CrossRef] 
59. Parcerisa, J.; Codony, R.; Boatella, J.; Rafecas, M. Triacylglycerol and phospholipid composition of hazelnut (Corylus avellana L.) lipid fraction during fruit development. J. Agric. Food Chem. 1999, 47, 1410-1415. [CrossRef]

60. Jalil, A.M.; Ismail, A. Polyphenols in cocoa and cocoa products: Is there a link between antioxidant properties and health? Molecules 2008, 13, 2190-2219. [CrossRef]

61. Miller, K.B.; Hurst, W.J.; Payne, M.J.; Stuart, D.A.; Apgar, J.; Sweigart, D.S.; Ou, B. Impact of alkalization on the antioxidant and flavanol content of commercial cocoa powders. J. Agric. Food Chem. 2008, 56, 8527-8533. [CrossRef] [PubMed]

62. Lipp, M.; Simoneau, C.; Ulberth, F.; Anklam, E.; Crews, C.; Brereton, P.; de Greyt, W.; Schwack, W.; Wiedmaier, C. Composition of genuine cocoa butter and cocoa butter equivalents. J. Food Compos. Anal. 2001, 14, 399-408. [CrossRef]

63. Bignami, C.; Cristofori, V.; Troso, D.; Bertazza, G. Kernel quality and composition of hazelnut (Corylus avellana L.) cultivars. Acta Hortic. 2005, 686, 477-484. [CrossRef]

64. Ebrahem, K.S.; Richardson, D.G.; Tetley, R.M.; Mehlenbacher, S.A. Oil content, fatty acid composition and vitamin E concentration of hazelnut varieties, compared to other types of nuts and oilseeds. Acta Hortic. 1994, 351, 685-692. [CrossRef]

65. Botta, R.; Gianotti, C.; Richardson, D.; Suwanagul, A.; Carlos, L.S. Hazelnut variety organic acids, sugars, and total lipid fatty acids. Acta Hortic. 1994, 351, 693-699. [CrossRef]

66. Machálková, L.; Hřivna, L.; Nedomová, S.; Jůzl, M. The effect of storage temperature on the quality and formation of blooming defects in chocolate confectionery. Potravinarstvo 2015, 9, 39-47. [CrossRef]

67. Thamke, I.; Dürrschmid, K.; Rohm, H. Sensory description of dark chocolate by consumers. LWT Food Sci. Technol. 2009, 42, 534-539. [CrossRef]

68. Yadav, P.; Pandey, J.P.; Garc, S.K. Biochemical changes during storage of chocolates. Int. Res. J. Biochem. Bioinform. 2011, 1, 242-247.

69. Gordon, M.H. Chapter 2-The development of oxidative randicity in foods. In Antioxidants in Food-Practical Applications; Pokorni, J., Yanishlieva, N., Gordon, M., Eds.; Woodhead Publishing Ltd.: Shaston, UK, 2001; pp. 7-21.

70. Fardelli, A. Effect of Storage Conditions on Hazelnuts (Corylus avellana L.) Quality. Ph.D. Thesis, Università degli Studi della Tuscia, Via Santa Maria in Gradi, Italy, 2008.

71. Lopez-Uriarte, P.; Bulló, M.; Casas-Agustench, P.; Babio, N.; Salas-Salvadó, J. Nuts and oxidation: A systematic review. Nutr. Rev. 2009, 67, 497-508. [CrossRef]

72. Subramaniam, P.J. Confectionery products. In The Stability and Shelf-Life of Food; Llcast, D., Subramaniam, P.J., Eds.; CRC Press: Boca Raton, FL, USA, 2000; pp. 221-248.

73. Lamuela-Raventòs, R.M.; Romero-Pérez, A.I.; Andrés-Lacueva, C.; Tornero, A. Review: Health Effects of Cocoa Flavonoids. Food Sci. Technol. Int. 2005, 11, 159-176. [CrossRef]

74. Othman, A.; Ismail, A.; Ghani, A.N.; Adenan, I. Antioxidant capacity and phenolic content of cocoa beans. Food Chem. 2007, 100, 1523-1530. [CrossRef]

75. Kornsteiner, M.; Wagner, K.H.; Elmadfa, I. Tocopherols and total phenolics in 10 different nut types. Food Chem. 2006, 98, 381-387. [CrossRef]

76. Bomba, P.C. Shelf life of chocolate confectionery products. In Shelf Life Studies of Foods and Beverages; Charalambous, G., Ed.; Elsevier: Amsterdam, The Netherlands, 1993; pp. 341-351.

77. Stauffer, M. The flavor of milk chocolate-Changes caused by processing. Manuf. Confect. 2000, 80, 113-118.

78. Seppanen, C.M.; Song, Q.; Csallany, A.S. The antioxidant functions of tocopherol and tocotrienol homologues in oils, fats, and food systems. J. Am. Oil Chem. Soc. 2010, 87, 469-481. [CrossRef]

79. Baccelloni, S. Recupero di Antiossidanti Naturali dai Sottoprodotti di Trasformazione Dell'industria Agroalimentare: Noccioli di Oliva (Olea europaea L.) Gusci e Perisperma di Nocciole (Corylus avellana L.). Ph.D. Thesis, Università degli Studi della Tuscia, Via Santa Maria in Gradi, Italy, 2006.

(C) 2019 by the authors. Licensee MDPI, Basel, Switzerland. This article is an open access article distributed under the terms and conditions of the Creative Commons Attribution (CC BY) license (http://creativecommons.org/licenses/by/4.0/). 